\title{
Cucumis melo MicroRNA Expression Profile During Aphid Herbivory in a Resistant and Susceptible Interaction
}

\author{
Sampurna Sattar, ${ }^{1}$ Yan Song, ${ }^{2,3}$ James A. Anstead, ${ }^{1}$ Ramanjulu Sunkar, ${ }^{3}$ and Gary A. Thompson ${ }^{1}$ \\ ${ }^{1}$ College of Agricultural Sciences, The Pennsylvania State University, University Park 16802, U.S.A.; ${ }^{2}$ Bioinformatics Core \\ Facility and ${ }^{3}$ Department of Biochemistry and Molecular Biology, Oklahoma State University, Stillwater 74078, U.S.A.
}

Submitted 28 September 2011. Accepted 22 February 2012.

\begin{abstract}
Aphis gossypii resistance in melon (Cucumis melo) is due to the presence of a single dominant virus aphid transmission (Vat) gene belonging to the nucleotide-binding site leucinerich repeat family of resistance genes. Significant transcriptional reprogramming occurs in $\mathrm{Vat}^{+}$plants during aphid infestation as metabolism shifts to respond to this biotic stress. MicroRNAs (miRNAs) are involved in the regulation of many biotic stress responses. The role of miRNAs was investigated in response to aphid herbivory during both resistant and susceptible interactions. Small RNA (smRNA) libraries were constructed from bulked leaf tissues of a $\mathrm{Vat}^{+}$melon line following early and late aphid infestations. Sequence analysis indicated that the expression profiles of conserved and newly identified miRNAs were altered during different stages of aphid herbivory. These results were verified by quantitative polymerase chain reaction experiments in both resistant $\mathrm{Vat}^{+}$and susceptible $\mathrm{Vat}^{-}$ interactions. The comparative analyses revealed that most of the conserved miRNA families were differentially regulated during the early stages of aphid infestation in the resistant and susceptible interactions. Along with the conserved miRNA families, 18 cucurbit-specific miRNAs were expressed during the different stages of aphid herbivory. The comparison of the miRNA profiles in the resistant and susceptible interactions provides insight into the miRNA-dependent post-transcriptional gene regulation in Vat-mediated resistance.
\end{abstract}

MicroRNAs (miRNA) are a class of non-protein coding RNA that plays an important role in regulating gene expression of protein-coding genes at the RNA level. miRNAs are typically 18 to 22 nucleotides (nt) in size and are the most abundant class of endogenous small RNA (smRNA) in both plants and animals. In plants, miRNAs are known to regulate different biological processes, including development, metabolism, defense against pathogens, and various types of biotic and abiotic stress responses (Katiyar-Agarwal and Jin 2010; Sunkar 2010). The regulatory role of plant miRNAs is manifested by its ability to bind to the target mRNA with near-perfect complementarity, thereby, causing post-transcriptional regulation of gene expression (Jones-Rhoades et al. 2006). Biogenesis of

Corresponding author: S. Sattar; Telephone: +1 814.863.5263; Fax: +1814 . 863.7905; E-mail: sus56@psu.edu

* The $\boldsymbol{e}$-Xtra logo stands for "electronic extra" and indicates that three supplementary tables and one supplementary figure are published online. plant miRNA takes place in the nucleus (Zhu 2008). The primary miRNA (pri-miRNA) transcripts range in size from approximately 100 to 1,000 nucleotides (nt) and are processed by a double-stranded (ds)RNA specific RNaseIII enzyme Dicerlike 1 (DCL1) into a mature miRNA and miRNA* duplex. The duplex is methylated by Hua Enhancer 1 and exported to the cytoplasm where it is incorporated into the Argonuate 1-containing RNA-induced silencing effector complex (RISC). The mature miRNA guides RISC to the target mRNA, resulting in cleavage and repression of the target gene expression (Mallory and Vaucheret 2010).

Plant miRNAs respond to different types of biotic stress. In Arabidopsis, miR393 was shown to play a role in bacterial pathogenesis by negatively regulating the transport inhibitor responsive 1 (TIR1) gene, which is involved in auxin signaling (Navarro et al. 2006). The expression of miR160 and miR167 are also altered during bacterial infections, and these miRNAs target members of the auxin-response factor (ARF) family of transcription factors that are involved in the auxin signaling pathway (Fahlgren et al. 2007). Plant miRNAs have been implicated in other biotic interactions such as fungal infections ( $\mathrm{Lu}$ et al. 2007b) and Turnip mosaic virus infection (He et al. 2008). Hewezi and coworkers (2008) found reduced susceptibility of Arabidopsis DCL and RNA-directed RNA polymerase (RdR) mutants to nematodes, implicating the involvement of smRNAs in plant-nematode interactions. In contrast, silencing $R d R I$ makes the Nicotiana attenuata more susceptible to insect herbivory by chewing insects (Pandey et al. 2008). Altered expression levels of genes involved in jasmonic acid (JA) and ethylene (ET) biosynthesis and reduced accumulation of JA in $R d R l$-silenced Nicotiana plants indicates that smRNAs are involved in defense signaling in response to insect herbivory (Pandey et al. 2008).

Aphid herbivory induces differential expression of genes encoding proteins belonging to the phytohormone signaling pathways that are involved in defense responses (Smith and Boyko 2007). Aphids activate plant defense signaling pathways dependent on both salicylic acid (SA) and JA signaling molecules (Kaloshian 2004; Moran et al. 2002). Recently, Anstead and co-workers (2010) have shown that ET-related genes are activated in response to cotton-melon aphid (Aphis gossypii) feeding in resistant and susceptible melon (Cucumis melo) lines. A. gossypii resistance in melon is mediated by the single, dominant virus aphid transmission (Vat) gene, which is a member of the coiled-coil nucleotide-binding site leucine-rich repeat subfamily of plant resistance genes (Dogimont et al. 2008). Vatmediated genetic resistance to aphids was identified in several sources of germplasm, including the Indian accession PI 371795 
that was subsequently introgressed into the susceptible aphid breeding line PMR 5 by eight successive backcrosses, followed by six generations of selfing to produce the nearly isogenic aphid resistant line AR 5 (McCreight et al 1984). Vat confers dual resistance against $A$. gossypii as well as several mosaic viruses transmitted by this aphid. Aphid resistance is exhibited as antixenosis (i.e., nonpreference of the aphids) and as antibiosis (i.e., reduction in aphid performance and fecundity) (Bohn et al. 1972), which significantly shortens the reproductive phase and life span of the aphids (Klingler et al. 1998). The Vat-mediated aphid resistance phenotype is expressed primarily in the phloem throughout all developmental stages in melon, although tissues along the pathway to the phloem could contribute to the resistance (Chen et al. 1996; Garzo et al. 2002; Kennedy and Kishaba 1977; Klingler et al. 1998).

A. gossypii infestation induces significant transcriptional reprogramming in melon plants with Vat-mediated resistance. Transcripts for several ET-responsive genes accumulate in response to A. gossypii feeding on a resistant melon line. Genes encoding transcription factors in a regulatory cascade associated with the ET signaling pathway are highly induced within the first $6 \mathrm{~h}$ after infestation during the resistant interaction (J. A. Anstead 2010). Several other transcription factor families that are associated with resistance such as NAC and bHLH are induced during aphid infestation in $\mathrm{Vat}^{+}$plants (J. Anstead, P. Samuel, N. Song, C. Wu, G. A. Thompson, and F. Goggin, unpublished data). Interestingly, many of these transcription factors are regulated by miRNAs: miR164 targets NAC transcription factors and miR393 regulates members of the bHLH family of transcription factors (Vidal et al. 2010). Expression profiles of smRNAs during different stages of A. gossypii infestation were studied to determine whether miRNAs are involved in regulating aphid-induced gene expression in nearly isogenic susceptible and resistant melon lines.

\section{RESULTS}

\section{Sequence analysis of the $C$. melo smRNA population in $\mathrm{Vat}^{+}$lines.}

Three smRNA libraries were generated from pooled leaf tissues of $\mathrm{Vat}^{+}$melon line AR 5 that were collected at different time points with and without aphid infestations and sequenced using an Illumina GAII analyzer. The control library yielded approximately 8 million raw reads, whereas the early and late library produced approximately 4.5 and 4.9 million reads, respectively. After removing the low-quality reads, adapter, and redundant sequences, there were about 7 million, 3.5 million, and 4.3 million unique sequences in the control, early, and late libraries, respectively (Table 1). Only those sequences of 18 to $30 \mathrm{nt}$ with a count of 10 or more were retained as unique sequences (Supplementary Fig. S1). In the three libraries combined, 45 to $50 \%$ of the total smRNA were $24 \mathrm{nt}, 11$ to $15 \%$ were $23 \mathrm{nt}, 8$ to $9.5 \%$ were $22 \mathrm{nt}$, and 10 to $12 \%$ were $21 \mathrm{nt}$ (Fig. 1). Endogenous siRNAs of $24 \mathrm{nt}$ are the most abundant smRNAs in some plants such as Arabi- dopsis, tomato, and, most recently, in another cucurbit, cucumber (C. sativus) (Martinez et al. 2011). In contrast, 24-nt smRNAs were not the predominant class identified from the melon smRNA transcriptome analyzed from Watermelon mosaic virus and Melon necrotic spot virus susceptible ('Tendril') and resistant ('T-111' and 'TGR-1551') cultivars (GonzalezIbeas et al. 2011). The total number of smRNA sequences identified from the two aphid-infested libraries was larger in the early and late libraries compared with the control library (Table 1). Among the conserved miRNAs found in those libraries, $75 \%$ were $21 \mathrm{nt}$, with the remaining $25 \%$ comprising 19,20 , and $22 \mathrm{nt}$, and only a small fraction ( 2 to $5 \%$ ) represented by 18 - to 20 -nt smRNAs.

\section{Identification of aphid-induced conserved miRNAs in the resistant interaction.}

To identify conserved miRNAs that accumulate in response to aphid herbivory on an aphid-resistant melon line, unique reads in the libraries were matched to the repository of plant miRNAs in miRBase using custom-written Perl script. Two mismatches were allowed during the sequence homology searches that resulted in the identification of 23 conserved miRNAs families in melon (Table 2). The total counts of conserved miRNAs were doubled in the late library when compared with the control, while the early library also showed a considerable increase (Table 1). Several conserved miRNAs were upregulated during aphid herbivory compared with the noninfested control library (Table 2). However, individual members of an miRNA family were not expressed uniformly in each of the libraries; some members were more abundant compared with others during the early and late stages of aphid infestation. The library counts of individual members in conserved miRNA families are documented in Supplementary Table S1.

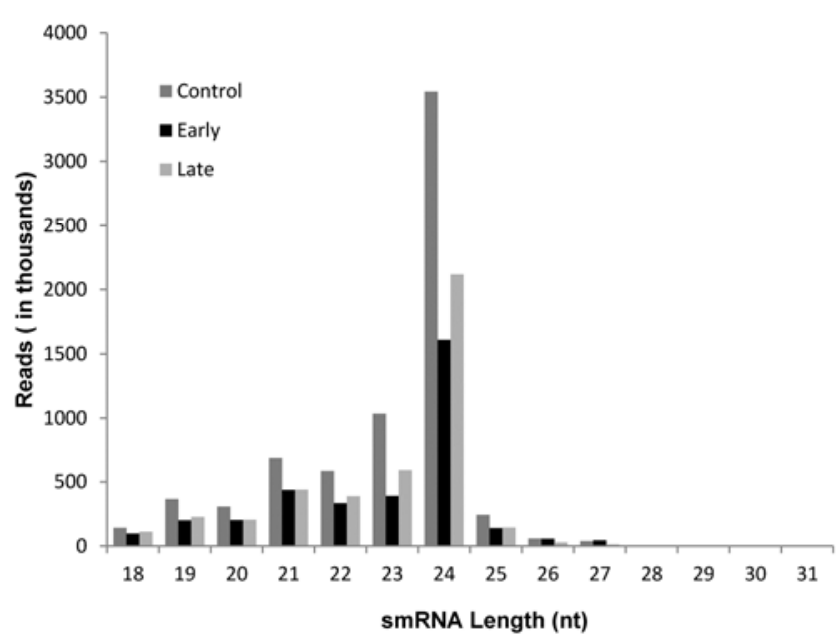

Fig. 1. Size distribution of small RNA (smRNA) sequences in control, early, and late melon libraries.

Table 1. Statistics of small RNA (smRNA) sequences from control, early, and late libraries

\begin{tabular}{lrrr}
\hline Statistics $^{\mathbf{a}}$ & Control & \multicolumn{1}{c}{ Early } & Late \\
\hline Raw reads & $8,026,527$ & $4,464,528$ & $4,886,463$ \\
Adaptors removed & $7,010,010$ & $3,526,290$ & $4,265,485$ \\
rRNA/tRNA exact matches removed & 114,154 & 93,012 & 105,953 \\
Match to known miRNAs & 822 & 1,458 & 864 \\
Match to known pumpkin smRNAs & 15,149 & 19,936 & 14,512 \\
Match to cucumber flower 454 EST database & 13,506 & 14,914 & 10,217 \\
Match to melon unigene database & 19,455 & 17,991 & 20,371 \\
\hline
\end{tabular}

${ }^{\mathrm{a}}$ miRNA $=$ microRNA and $\mathrm{EST}=$ expressed sequence tag. 


\section{Identification of cucurbit specific miRNAs and novel miRNAs.}

Following the identification of conserved miRNA, the remaining unique sequences in the library were searched for the presence of novel miRNAs. Melon whole-genome sequencing is still in progress; therefore, the melon expressed sequence tag (EST) collection (version 3.0) was used for this analysis. The closest relative of melon to have a complete genome available is cucumber, which was also used to identify cucurbit-specific miRNAs. The pumpkin (Cucurbita maxima) smRNA database was also used to search for smRNAs specific to this family. Unique sequences that did not match with conserved plant miRNAs were matched to these databases using the miRDeep algorithm. This algorithm uses the probabilistic model of the miRNA biogenesis to score the compatibility and the position of the sequence smRNA to the secondary structure of the miRNA precursor (Friedlander et al. 2008). Though miRDeep was originally described for identifying animal miRNAs, it has since been modified to successfully identify plant miRNAs (Yang et al. 2011). Animal miRNAs have a shorter precursor length compared with plant miRNA precursors that vary from 100 to $1,000 \mathrm{bp}$. Precursor sequences of $300 \mathrm{bp}$ were processed into stem-loop structures to identify novel candidate miRNAs in melon. This analysis identified 18 putative, cucurbit-specific miRNAs. Most of the miRNAs were $21 \mathrm{nt}(42 \%)$, followed by $22 \mathrm{nt}(26 \%), 23 \mathrm{nt}(16 \%), 24 \mathrm{nt}(10 \%)$, and $20 \mathrm{nt}(5.2 \%)$. Of these, 5 (Cme-miR-2, Cme-miR-4, Cme-miR-5, Cme-miR-8, and Cme-miR-9) were melon specific, while the remaining 13 sequences were identified from both melon and pumpkin smRNA databases (Table 3). Interestingly, none of the novel miRNAs identified in cucumber (Martinez et al. 2011) were represented in any of the three melon libraries, indicating that those reported miRNAs are species specific. The secondary structures predicted for each of the novel candidate melon-specific miRNAs are shown in Figure 2. Some of the sequences from the three libraries matched to the sequences in the pumpkin smRNA database. Approximately $0.21 \%$ of the control library population, $0.56 \%$ of the early library, and $0.34 \%$ of the late library matched to the smRNA sequences from pumpkin (Table 1).

Table 2. Conserved microRNA (miRNA) families and their predicted target genes in melon

\begin{tabular}{|c|c|c|c|c|c|c|}
\hline \multirow[b]{2}{*}{ Family } & \multirow[b]{2}{*}{ Sequence } & \multicolumn{3}{|c|}{ Normalized library requency $^{a}$} & \multicolumn{2}{|r|}{ Target $^{\mathrm{b}}$} \\
\hline & & Control & Early & Late & Conserved & Putative \\
\hline $\operatorname{miR} 156$ & AUGACAGAAGAUAGAGAGCAC & 30,006 & 16,255 & 22,676 & SPB protein & MU29917 (SPB protein) \\
\hline $\operatorname{miR} 157$ & UGACAGCAGAUAGAGAGCAC & 781.8 & 304.9 & 216.5 & SPB protein & MU34102 (SPB protein) \\
\hline miR159 & AUUGGAUUGAAGGGAGCUCUA & 231.2 & $1,056.2$ & 100.8 & MYB protein & $\begin{array}{l}\text { MU39994, MU40740 } \\
\text { (unknown) }\end{array}$ \\
\hline $\operatorname{miR} 160$ & AGCCUGGCUCCCUGUAUGCCA & 0 & 20.9 & 2.4 & ARF & MU38981 (ARF) \\
\hline $\operatorname{miR} 162$ & UCGAUAAACCUCUGCAUCCAA & 1.92 & 0 & 2.69 & DCL-1 & $\begin{array}{l}\text { MU30859, MU41038 } \\
\text { (unknown) }\end{array}$ \\
\hline $\operatorname{miR} 164$ & UGGAGAAGCAGGGCACGUGCA & 30.71 & 225.5 & 312.5 & NAC domain protein & $\begin{array}{l}\text { MU22717 (NAC domain } \\
\text { protein, SORBIDRAFT) }\end{array}$ \\
\hline $\operatorname{miR} 165$ & UCGGACCAGGCUUCAAUCCCC & 10.83 & 187.5 & 60.2 & $\begin{array}{l}\text { HD-Zip transcription } \\
\text { factors }\end{array}$ & No target identified \\
\hline $\operatorname{miR} 166$ & AACGGACCAGGCUUCAUUCCCC & 9,735 & 178,641 & 33,844 & $\begin{array}{l}\text { HD Leucine Zipper III } \\
\text { transcription factors }\end{array}$ & No target identified \\
\hline $\operatorname{miR} 167$ & ACAAGCUGCCAGCAUGAUCUAA & $3,135.7$ & 4019 & 11,826 & $\mathrm{ARF}$ & MU26857 (ARF3) \\
\hline $\operatorname{miR} 168$ & ACGCAUGGUGCAGGUCGGGAA & $2,224.4$ & 25,433 & $6,506.1$ & AGO1 & No target identified \\
\hline $\operatorname{miR} 169$ & CAGCCAAGGAUGAAUUGCCGG & 453.4 & 571.4 & $1,748.1$ & $\begin{array}{l}\text { CCAAT binding factor- } \\
\text { HAP2-like proteins }\end{array}$ & $\begin{array}{l}\text { MU31362 } \\
\text { (no matches in BLAST) }\end{array}$ \\
\hline $\operatorname{miR} 171$ & UGAUUGAGCCGCGCCAAUAUC & 63.3 & 115.1 & 431.9 & $\begin{array}{l}\text { Scarecrow proteins- } \\
\text { transcription factors }\end{array}$ & $\begin{array}{l}\text { MU36464 } \\
\text { (no matches in BLAST) }\end{array}$ \\
\hline $\operatorname{miR} 172$ & AGAAUCUUGAUGGUGCUGCAU & $6,951.9$ & $1,357.3$ & 10,122 & Apetala 2 (AP2) proteins & $\begin{array}{l}\text { MU29652, MU31408, } \\
\text { MU33447 (AP2 like protein) }\end{array}$ \\
\hline $\operatorname{miR} 2111$ & UAAUCUGCAUCCUGAGGUUUC & 53.4 & 204.3 & 94.4 & Unknown & $\begin{array}{l}\text { MU34470 } \\
\text { (Phospholipid/glycerol acyl } \\
\text { transferase protein) }\end{array}$ \\
\hline $\operatorname{miR} 2911$ & UCCGGGGGACGGACUGGGA & 0 & 7.9 & 3.1 & Unknown & $\begin{array}{l}\text { MU27177 (CytP450 like TBP, } \\
\text { senescence associated protein) }\end{array}$ \\
\hline $\operatorname{miR} 390$ & CAGCUCAGGAGGGAUAGCGCC & 0 & 43.4 & 9.78 & Unknown & $\begin{array}{l}\text { MU29926, MU39453 } \\
\text { (no matches in BLAST) }\end{array}$ \\
\hline $\operatorname{miR} 393$ & UCCAAAGGGAUCGCAUUGAUC & 23.59 & 10.1 & 22.2 & $\begin{array}{l}\text { F-box proteins and bHLH } \\
\text { transcription factors }\end{array}$ & $\begin{array}{l}\text { MU21869 (Transport inhibitor } \\
\text { responsive factor, AFB2 } \\
\text { [f-box protein]) }\end{array}$ \\
\hline miR394 & UUGGCAUUCUGUCCACCUCC & 20.77 & 11.1 & 28.38 & F-box proteins & No target identified \\
\hline miR395 & CUGAAGUGUUUGGGGGAACUC & 1.63 & 5.39 & 5.87 & ATP sulphurylases & MU24817 (ATP sulphurylase) \\
\hline $\operatorname{miR} 396$ & AUCCACAGCUUUCUUGAACUG & 623.4 & 605.3 & $1,367.8$ & Growth regulating factors & $\begin{array}{l}\text { MU41399, MU21674 (no } \\
\text { match, mitochondria import } \\
\text { receptor subunit TOM20) }\end{array}$ \\
\hline $\operatorname{miR} 397$ & CAUUGAGUGCAGCGUUGAUGU & 24.9 & 15.9 & 73.2 & Laccases and $\beta-6$ tubulin & $\begin{array}{l}\text { MU40742 (laccase-1a,diphenol } \\
\text { oxidase) }\end{array}$ \\
\hline $\operatorname{miR} 398$ & UGUGUUCUCAGGUCGCCCCUG & 1.78 & 7.61 & 5.1 & $\begin{array}{l}\text { Copper superoxide dis- } \\
\text { mutases and cytochrome } \\
\text { C-oxidase subunit }\end{array}$ & \\
\hline $\operatorname{miR} 408$ & CUGCACUGCCUCUUCCCUGGC & 0 & 9.5 & 0 & Stress-related miRNA & $\begin{array}{l}\text { MU25436, MU24829 (predicted } \\
\text { protein, 2CBP cucumber basic } \\
\text { protein, chain A blue } \mathrm{Cu} \text { protein) }\end{array}$ \\
\hline miR894 & CGAUUCACGUCGGGUUCAC & 23.6 & 279.5 & 73.4 & Unknown & No target identified \\
\hline
\end{tabular}

${ }^{\mathrm{a}}$ Normalized frequency $=($ number of reads/total clone counts in the library $) \times 10^{6}$.

${ }^{\mathrm{b}}$ Conserved target in Arabidopsis and putative target in melon and its annotation. SPB $=$ Squamosa promoter binding and ARF $=$ auxin response factor. 
Expression profile of conserved miRNA during different stages of aphid herbivory

in resistant and susceptible melon lines.

The bioinformatic analysis of the smRNA libraries revealed that the expression of most conserved miRNAs increased during the early and late stages of aphid herbivory. Quantitative real-time polymerase chain reaction (qPCR) experiments were performed to validate the sequencing-based profiling data. The deep sequencing data revealed that individual members of a conserved family were differentially expressed during the early and late stages of aphid herbivory. The library data showed increases of miR160, miR164, miR165, miR166, miR167, miR169, miR2111, miR2911, miR390, miR393, miR396, miR397, miR398, miR408, and miR894 during early

Table 3. Cucurbit-specific microRNAs (miRNAs) and novel miRNAs identified from melon

\begin{tabular}{|c|c|c|c|c|c|c|c|}
\hline \multirow[b]{2}{*}{ Cme-miRNA $^{a}$} & \multirow[b]{2}{*}{ Length } & \multirow[b]{2}{*}{ Novel miRNA sequence } & \multicolumn{3}{|c|}{ Normalized library frequency } & \multirow[b]{2}{*}{ Target } & \multirow[b]{2}{*}{ Annotation } \\
\hline & & & Control & Early & Late & & \\
\hline miR-1 & 24 mer & GGAAUGUUGUCUGGCUCGAGGACA & 10.2 & 0 & 0 & No target & \\
\hline miR-2 & 23 mer & AGUCAAAUCUAAACGAUCGUGUA & 1.7 & 0 & 0 & $\begin{array}{l}\text { MU31433, } \\
\text { MU35904 }\end{array}$ & No hits \\
\hline miR-3 & 21 mer & GCGUAUGAGGAGCCAUGCAUA & 77.3 & 0 & 0 & No target & \\
\hline miR-4 & 23 mer & GUUGACAGAAGAGAGUGAGCACA & 38.6 & 0 & 136 & $\begin{array}{l}\text { MU34102, } \\
\text { MU29917 }\end{array}$ & $\begin{array}{l}\text { Liguleless-1 protein, } \\
\text { POPTRDRAFT_576281 }\end{array}$ \\
\hline miR-5 & 22 mer & UGAGCCAAGAAGACUUGCCGGC & 5.1 & 0 & 36.5 & No target & \\
\hline miR-6 & 21 mer & GCGUAUGAGGAGCCAAGCAUA & 4.7 & 0 & 41 & No target & \\
\hline miR-7 & 20 mer & CGACGUCGCGAGAAGUCCAU & 3.1 & 0 & 0 & MU21621 & Unknown protein \\
\hline miR-8 & 24 mer & GUUUGGAAUGUGCGAGAUGUGUGC & 6.5 & 0 & 0 & No target & \\
\hline miR-9 & 22 mer & AGGGCUUCUCUCCAUUGGCAGG & 0 & 67.5 & 0 & No target & \\
\hline miR-10 & 22 mer & GCUCACUUCUCUUUCUGUCAGU & 0 & 24.4 & 0 & No target & \\
\hline $\operatorname{miR}-11$ & 21 mer & GUGACAGAAGAGAGUGAGCAC & 0 & 258.3 & 0 & MU29917 & $\begin{array}{c}\text { POPTRDRAFT_755123, } \\
\text { POPTDRAFT_576281 }\end{array}$ \\
\hline $\operatorname{miR}-12$ & 21 mer & UUCCAUCUCUUGCACACUGGA & 0 & 4.8 & 0 & MU41094 & $\begin{array}{l}\text { F-box family protein, } \\
\text { ubiquitin protein ligase }\end{array}$ \\
\hline miR-13 & 21 mer & ACCGGUCGGCUCGUCCCUUUU & 0 & 3.7 & 0 & No target & \\
\hline miR-14 & 21 mer & AUCUCGGACCAGGCUUCAUUC & 0 & 19.3 & 0 & No target & \\
\hline miR-15 & 22 mer & CGGCAAGUCGUCCUUGGCUACC & 0 & 0 & 251 & No target & \\
\hline miR-16 & 21 mer & GGGAUAUUGGUGCGGUUCAAU & 0 & 0 & 5.9 & No target & \\
\hline miR-17 & 22 mer & GCUCACUUCUCUUUCUGUCAGC & 0 & 0 & 34.7 & No target & \\
\hline miR-18 & 23 mer & UGAAGCUGCCAGCAUGAUCUGAA & 0 & 0 & 71 & No target & \\
\hline
\end{tabular}

${ }^{a}$ miRNAs in bold are novel miRNAs identified only in melon.

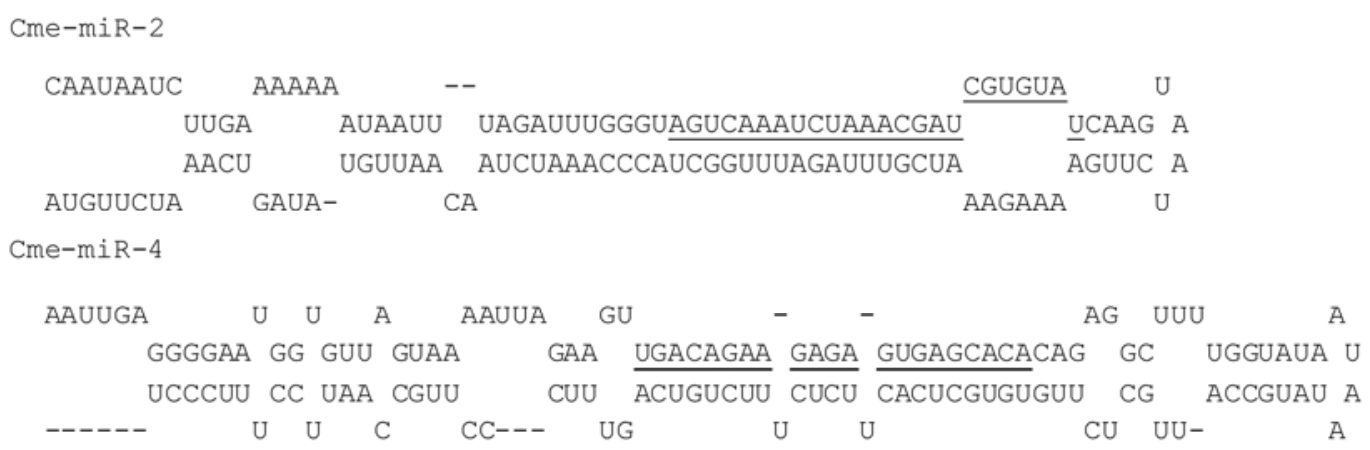

Cme-miR-5

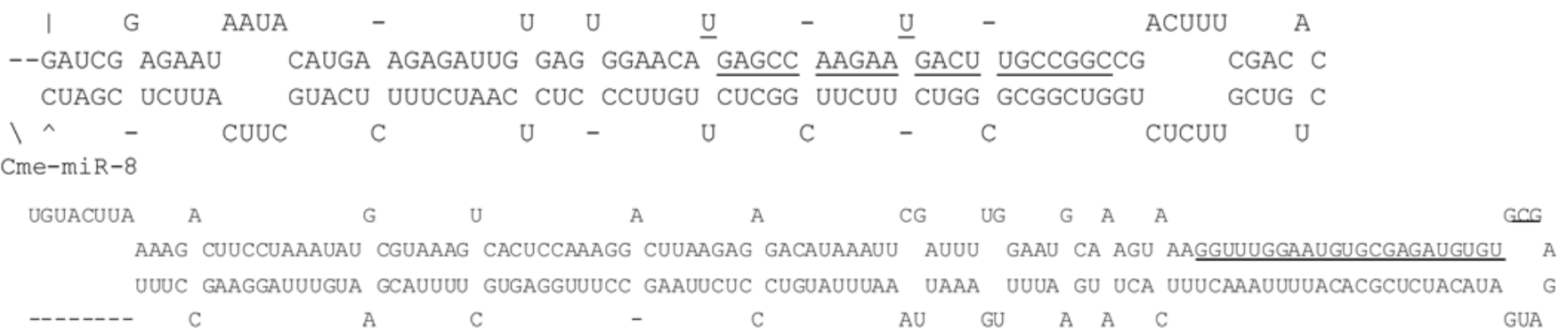

Cme-miR-9

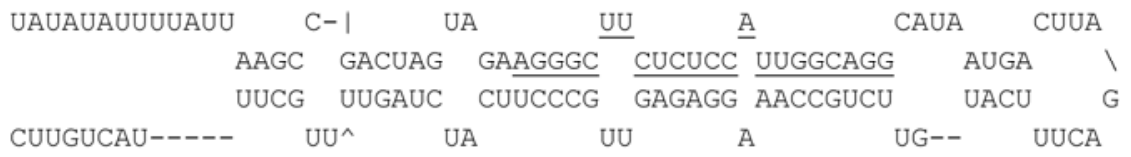

Fig. 2. Predicted secondary structure of primary microRNA (miRNA) transcripts of novel miRNAs. 
stages of aphid feeding, and qPCR data showed a similar trend (Fig. 3A). Following Bonferroni corrections for multiple comparisons of the qPCR data, miR166 and miR2911 showed significant $(P \leq 0.002)$ upregulation, providing strong statistical support for their differential expression during late stages of aphid infestations in AR 5. Support for differential upregulation of the other miRNA families was not as strong, because these families showed significance at $P \leq 0.05$ without Bonfer-
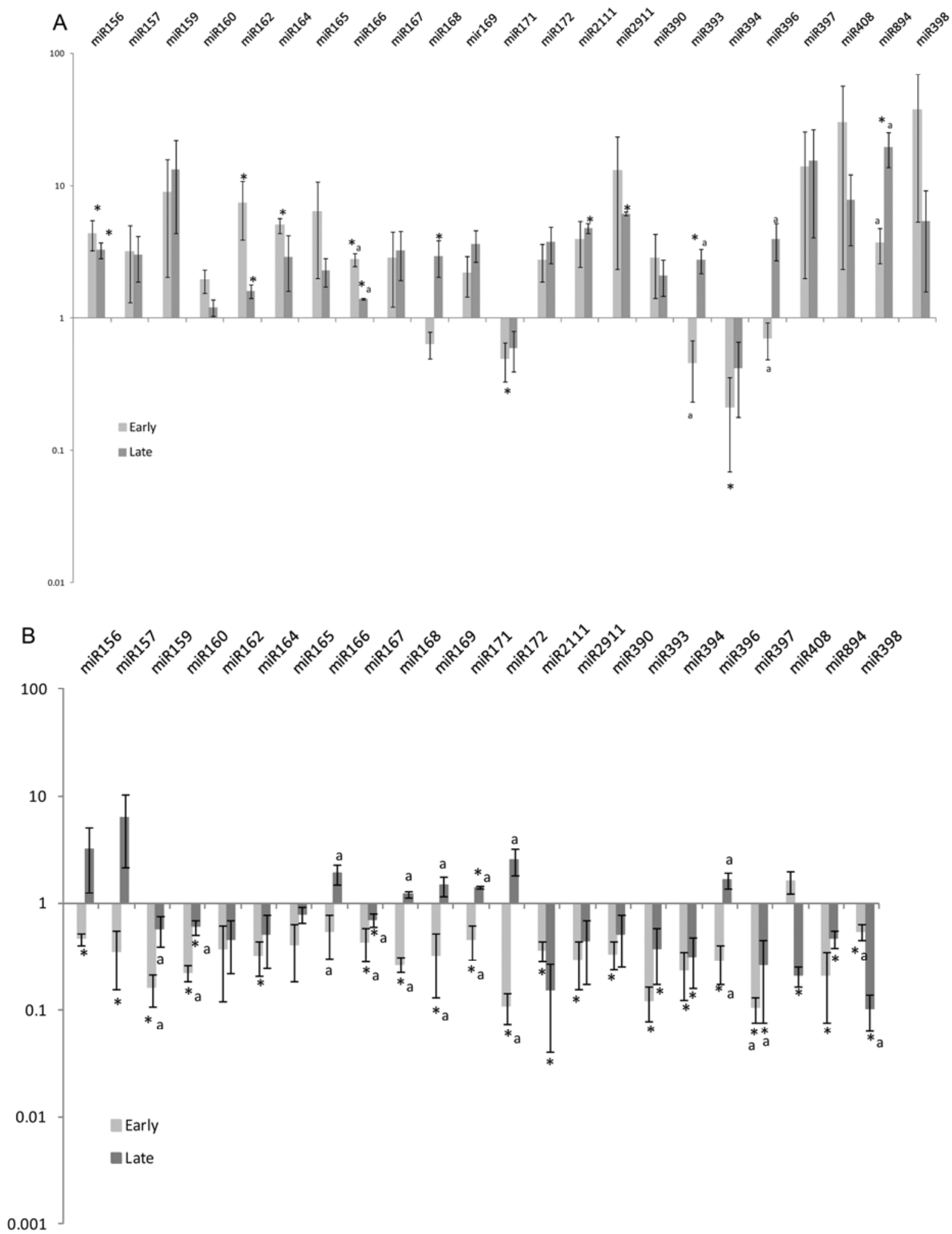

Fig. 3. Expression profiling of conserved microRNA (miRNA) differentially expressed in melon leaf tissues at different stages of aphid infestation. Mature miRNA expression level was estimated by quantitative real-time polymerase chain reaction using RNA isolated from aphid-infested and uninfested leaf tissue collected at early $(2,4$, and $6 \mathrm{~h})$ and late $(8,10$, and $12 \mathrm{~h})$ post aphid infestation. Expression levels were normalized using profilin as an internal reference gene. Fold changes were calculated using Pfaffl's method and represent change in the expression level of miRNA family relative to control uninfested leaf. Data are averages of three independent replicates \pm standard error of mean. Significance between control and aphid infestation was determined by Student's $t$ test, and is represented by * $(P \leq 0.05)$. Significance between the two time points post aphid infestation is represented as "a" $(P \leq 0.05)$. A, Expression profile during resistant interaction in the virus aphid transmission (Vat) gene $\mathrm{Vat}^{+}$melon line. B, Expression profile during susceptible interaction in $\mathrm{Vat}^{-}$melon line. 
roni corrections. The statistical analysis, conducted to reduce the frequency of type I errors (false positives), revealed significance for two conserved miRNA families. However, the overall expression trends revealed by the qPCR data are useful to develop additional hypotheses for the potential involvement of a broader range of miRNA families in aphid resistance. At this point, hypothesis development is the most important aspect of this research and, therefore, type I errors (false positives) are more acceptable than type II errors (false negatives). The qPCR expression data for miR156/miR157, miR162, miR168, and miR171 did not correspond to the library counts. To validate the library data and qPCR data, smRNA blots were performed for some of these miRNAs. smRNA blot analysis confirmed the qPCR expression pattern for miR156, miR157, miR159, and miR162, showing upregulation during the early stages of aphid herbivory (Fig. 4). The expression of miR156, miR162, and miR159 was shown to be downregulated during late stages of aphid herbivory by smRNA blots in accordance with the library data. Although miR395 also appears to increase in response to aphid feeding as revealed by the counts in three different libraries, miR395 expression could not be detected by the qPCR assay. smRNA blots detected two different miR395 sizes with distinct signals at 21 and 24 nt (Fig. 4) that were induced following aphid feeding.

The design and construction of the smRNA libraries in the $\mathrm{Vat}^{+}$melon line AR 5 only addresses the resistant interaction. Expression profiles of the conserved miRNAs between aphidresistant and -susceptible melon lines were compared by qPCR during the early and late stages of aphid infestation in the nearly isogenic, susceptible melon line PMR 5. In the susceptible interaction, 22 conserved miRNAs were downregulated in the early response to aphid infestation, whereas only miR408 was upregulated during the early stage and eight miRNAs (miR156, miR157, miR166, miR168, miR169, miR171, miR172, and miR396) were upregulated during the late stages of aphid herbivory (Fig. 3B). Following Bonferroni corrections, five miRNA families (miR159, miR160, miR172, miR168, miR393, and miR397) showed significant $(P \leq 0.002)$ downregulation during early stages and two families (miR408 and miR398) during the late stages of aphid infestation in PMR 5.

\section{Expression pattern of novel melon-specific miRNAs in the resistant and susceptible line.}

The expression profiles of novel melon-specific miRNAs identified by miRDeep were analyzed using low molecular weight (LMW) RNA isolated from AR 5 and PMR 5 by stemloop qPCR. In the resistant melon line AR 5, the expression of Cme-miR-2, Cme-miR-4, Cme-miR-5, Cme-miR-8, and CmemiR-9 did not show any significant change during both the early and late stages of aphid herbivory (Fig. 5A). The expression of these novel miRNAs was also studied in the susceptible line PMR 5, where Cme-miR-2, Cme-miR-8, and Cme-miR-9

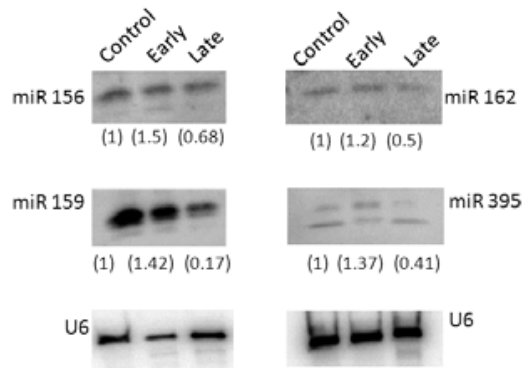

Fig. 4. Small RNA blots of microRNA (miRNA) families miR156, miR159, miR162, and miR395 expressed during early and late stages of aphid herbivory in resistant interaction. U6 was used for normalization. were significantly $(P \leq 0.05)$ downregulated during early stages of aphid infestation. Significant changes in the expression of these novel miRNAs between early and late stages of infestation were not observed.

\section{Prediction of miRNA targets and their validation by $5^{\prime}$ rapid amplification of cDNA ends (RACE).}

Plant miRNAs, unlike their animal counterparts, bind to their targets with near-perfect complementarity (Jones-Rhoades and Bartel 2004; Rhoades et al. 2002). This unique characteristic of plant miRNAs has been used to predict their mRNA targets. The targets of the conserved and the cucurbit-specific miRNAs were predicted by in silico analysis using the melon smRNA target identification tool. The predicted targets in melon are summarized in Tables 2 and 3. Most of the conserved plant miRNA target genes have been validated in Arabidopsis, rice, and poplar. Several putative target genes were identified in this study (Table 2), and a new target gene for miR398 was identified in melon. In Arabidopsis and rice, miR398 targets members of the copper superoxide dismutase (CSD) gene family. In melon, miR398 is predicted to target the gene represented by the melon EST MU25081; the miRNA-guided cleavage of MU25081 was validated by 5' RACE (Fig. 6). MU25081 is annotated as a homolog of Zea mays Uclacyanin-2 (UCC2) and

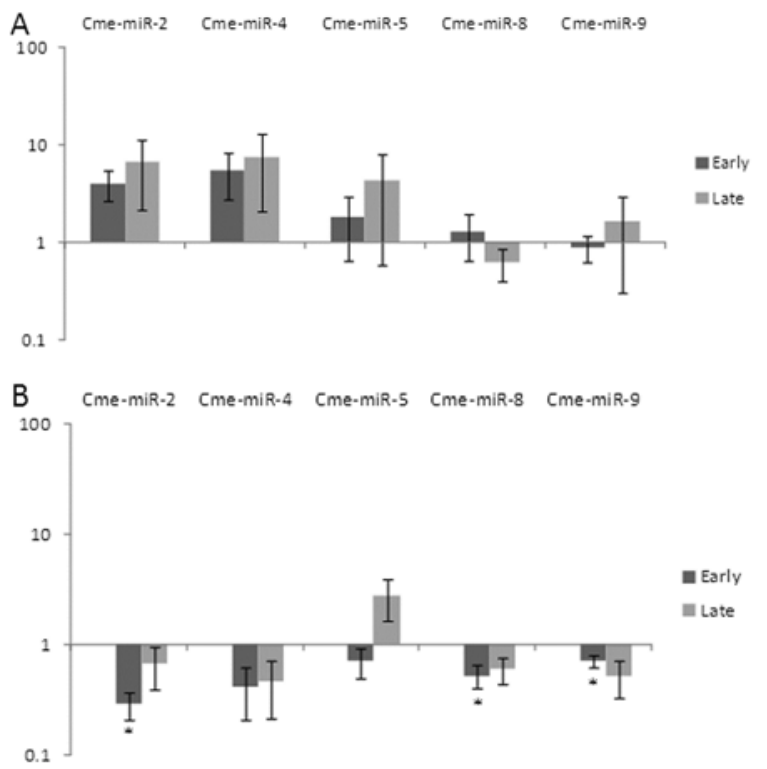

Fig. 5. Expression profile of the candidate novel microRNAs (miRNAs) during a resistant interaction. Mature miRNA expression level was estimated by quantitative real-time polymerase chain reaction using RNA isolated from aphid-infested and uninfested leaf tissue collected at early $(2,4$, and $6 \mathrm{~h}$ ) and late $(8,10$, and $12 \mathrm{~h}$ ) post aphid infestation. Expression levels were normalized using profilin as an internal reference gene. Fold changes were calculated using Pfaffl's method and represent change in the expression level of miRNA family relative to aphid-uninfested leaf as control. Data are averages of three independent replicates \pm standard error of mean. Significance between control and aphid infestation was determined by Student's $t$ test, and is represented by $*(P \leq 0.05)$. A, Novel miRNAs expressed in virus aphid transmission (Vat) gene $\mathrm{Vat}^{+}{ }^{+}$resistant line B, Vat $^{-}$ susceptible line of Cucumis melo.

\section{$\downarrow 17 / 30$ \\ 5 ' AAAATCTGCAAGCGGCGTCTGAGAACACACGCGTACAGA 3' MU25081 (UCC2) $x|| x|||||0|||||||||||$ 3 ' GUCCCCGCUGGACUCUUGUGU 5 ' miR398}

Fig. 6. Target validation of miR398 in melon. A $5^{\prime}$ rapid amplification of cDNA ends analysis was carried out for the predicted target gene. The $5^{\prime}$ end of the cleavage product is indicated by arrow. Target expressed sequence tag sequence is shown at the top of the microRNA sequence. 
the closest Arabidopsis homolog, AT2G26720.1, belongs to the same cupredoxin superfamily of proteins (Khan et al. 2007). Expression profiling in the resistant melon line revealed that miR398 was the most abundant miRNA during early stages of aphid feeding (Fig. 3A) and was downregulated in the susceptible interaction. Predicted targets could not be identified for miR159, miR165, miR166, miR168, miR394, and miR894, which is possibly due to the limited coverage of the melon EST library.

\section{DISCUSSION}

smRNAs are important gene regulators in controlling plant responses to biotic and abiotic stress. In this study, three smRNA libraries were generated from the aphid-resistant melon line AR 5 to compare smRNA expression in noninfested plants with responses that occur during two temporal stages during aphid herbivory. The rationale for choosing these two time points, representing early and late stages of aphid herbivory, is based on the distinctive pattern observed during the electrical penetration graph (EPG) studies in the nearly isogenic melon lines AR 5 and PMR 5. The EPG data revealed prolonged sieve-element probing and salivation accompanied by a significant inhibition of the sustained sap ingestion during the first $3 \mathrm{~h}$ of the interaction between A. gossypii and the resistant line AR 5 (Klingler et al. 1998). Sieve-tube sap ingestion quantified indirectly as rates of honeydew excretion decreased 10 -fold on AR 5 and was delayed by $6 \mathrm{~h}$ compared with the susceptible plants during a 12-h period (Klingler et al. 1998). Transcriptional studies revealed that expression of genes in the ET signaling pathway were differentially regulated between 6 and $24 \mathrm{~h}$, with gene induction occurring at $6 \mathrm{~h}$ compared with the 24-h time point. Thus, it appears that significant physiological events impacting resistance occur within the first $6 \mathrm{~h}$ of aphid infestation. In this study, pooled leaf tissues were collected from 2, 4, and $6 \mathrm{~h}$ post aphid infestation to represent the early stages. Similarly, leaf tissues collected from 8, 10, and $12 \mathrm{~h}$ were pooled together to represent the late stages of aphid infestation. Pooling of the leaf tissues provided maximum coverage for each stage of the interaction.

Twenty-three families of conserved plant miRNAs were identified from the three libraries (Table 2), and their expression patterns in response to aphid herbivory were studied in detail (Fig. 3). The numbers of conserved miRNAs in the libraries provided evidence that all of the conserved miRNA families were differentially regulated during the early and late phases of aphid herbivory in the resistant interaction (Table 2). These data are compatible with the results obtained from the EPG analyses of AR 5, where it was shown that aphids display prolonged probing behaviors prior to sustained sap ingestion (Klingler et al. 1998). Salivation that occurs during prolonged probing at the early stages of aphid infestation could initiate a resistance response by the plant and, in turn, trigger the induction of miRNAs and other smRNAs (Table 1). This agrees with the observations that $N$. attenuata mutants lacking $R d R l$ produce decreased amounts of smRNAs and are more susceptible to chewing insect attack (Pandey et al. 2008), suggesting that defense responses are regulated by smRNAs. Similarly, the susceptible melon line ( $\left.\mathrm{Vat}^{-}\right)$also showed an overall downregulation in the conserved miRNA population. This comparison yielded contrasting observations with respect to miRNAs expression or regulation in aphid resistant and susceptible lines during herbivory (Fig. 3). The $\mathrm{Vat}^{+}$interaction showed an overall trend toward enhanced miRNA expression, whereas the susceptible interaction showed downregulation of miRNAs. The opposite trends in these lines could be due to differences in miRNA transcription or biogenesis in the nearly isogenic susceptible and resistant melon lines during aphid feeding. The key components of the miRNA and siRNA biogenesis pathway are DCL proteins. Recently, Ahmadovichbozorov and coworkers (2012) have reported the regulatory role of different DCL proteins during Manduca sexta herbivory in N. attenuate. It will be of interest to identify the melon orthologues of the enzymes involved in the smRNA biogenesis pathway and study their expression profile in the susceptible and resistant melon lines to verify their role in the resistance mechanism.

A snapshot of the total smRNA population revealed that smRNA biology of the resistant $\mathrm{Vat}^{+}$melon line was distinctive from the susceptible Vat $^{-}$melon line. To understand the detailed role of these smRNAs in the resistance mechanism, specific miRNAs that are candidates for resistance were screened and further analyzed. Several stress-responsive miRNAs including miR160, miR164, miR167, miR393, miR395, miR398, and miR408, were identified in the melon libraries and their expression profile analyzed in the resistant and susceptible interactions (Fig. 3). The putative targets of these miRNAs were predicted by in silico analysis. Melon miR164 targets NAC6, which is a member of the NAC transcription factor family (Table 2). Barley NAC6 is a positive regulator of transcription involved in early defense against powdery mildews (Micali et al. 2008). Another group of miRNAs (miR160, miR167, and miR393) have well-established regulatory functions in bacterial resistance in Arabidopsis (Navarro et al. 2006). These miRNAs target the ARF family of genes and the TIR1 gene in Arabidopsis and are predicted to target ARF 2 and 3 and TIR1 genes in melon (Table 2). Kuśnierczyk and coworkers (2007) reported that several genes belonging to the auxin biosynthesis pathway were induced following green peach aphid (Myzus persicae) infestation in different eco-types of Arabidopsis. The significant downregulation of miR160, miR167, and miR393 in the susceptible melon line PMR 5 during both early and late stages of aphid feeding indicates a role for auxin-mediated gene regulation in the host-aphid interaction. Another stress responsive miRNA miR395 targets ATP-sulfurylases (ATPS) in Arabidopsis (Jones-Rhodes and Bartel 2004) and is predicted to have the same target in melon. The library data indicated accumulation of miR395 during aphid infestation during the resistant interaction. miR395 could not be detected by qPCR but two distinct signals were reported from the smRNA blots, which were differentially regulated during the early and later stages of the aphid infestation. ATPS is the first enzyme in the sulfate assimilation pathway and is sensitive to glutathione concentrations: ATPS activity is reduced in the presence of higher amounts of exogenous glutathione (Yi et al. 2010). Glutathione is an important antioxidant and plays a major role in plant defense by participating in reactive oxygen species signaling. Zechmann and associates (2009) have shown that the level of glutathione and its precursor cysteine is enhanced at the site of stylet penetration during black bean aphid (A. fabae) infestation in sugar beet. Glutathione synthase (GSH2) was also induced in Arabidopsis by green peach aphid adults and nymphs after $48 \mathrm{~h}$ of feeding (Kempema et al. 2007). It will be interesting to explore the role of miR395 in glutathione regulation via ATPS in response to A. gossypii infestation in melon.

Novel target genes were predicted for a number of miRNA families (miR169, miR171, miR2111, miR2911, miR396, miR398, and miR408) in melon (Table 2). A homolog of $Z$. mays UCC2 was confirmed as the target of melon miR398 by 5' RACE (Fig. 6). In Arabidopsis, miR398 targeted CSD1 and CSD2 genes and copper-chaperone for superoxide dismutase (Li et al. 2010; Sunkar et al. 2006). UCC2 is a glycosylphosphatidylinositol-anchored protein, which is a member 
of the family of copper-binding proteins involved in singleelectron transfer during redox reactions. A homolog of a putative senescence-associated gene (SAG) from Pisum sativum is the predicted target of miR2911. SAG are known to play a role in plant responses to aphid feeding. Increased SAG expression in response to $M$. persicae feeding in Arabidopsis was reported (Pegadaraju et al. 2005). Premature senescence of the aphid-infested leaf is induced in plants as part of the programmed degradation of cellular components and contributes to defense against the generalist insect $M$. persicae in Arabidopsis (Pegadaraju et al. 2005). It has been reported that aphid infestation changes the resource allocation in the host plant, leading to increased flow of nutrients to the insect-infested tissue due to the creation of a strong sink in those tissues (Girousse et al. 2005); however, Pegadaraju and co-workers (2005) have proposed that premature leaf senescence of the aphid-infested leaves inhibits the ability of the aphids to redirect the flow of resources and, thus, can be of benefit to the host plant. Upregulation of melon $S A G-21$ was reported by Anstead and associates (2010) in both resistant and susceptible interactions during the early stages $(6 \mathrm{~h})$ of aphid infestation. Another predicted target of melon miR2911 is a cytochrome P450 gene. Although an extremely large and diverse family, cytochrome P450s have well-established roles in wound healing and insect resistance (Noordermeer et al. 2001). In addition to the conserved targets, conserved miRNAs appear to target additional genes in different plant species (Lindow and Krogh 2005). The completion and release of the melon genome sequence will aid in the validation of predicted targets and should lead to the identification of additional target genes.

Several novel miRNAs were identified from the melon libraries. Eighteen putative cucurbit-specific miRNAs were identified from the melon libraries, five of which were specific to melon. In contrast to the conserved miRNA families that have multiple family members, the cucurbit-specific miRNAs are represented by a single member. Potential precursors were identified and a predicted fold-back structure was generated for each of these miRNAs. In silico target prediction revealed targets for only a subset of these cucurbit-specific miRNAs (Table 3). Cme-miR-4 was predicted to target ligueless-1 protein, a transcription factor homologous to Squamosa promoterbinding protein and POPTDRAFT_576281 from poplar (Populus tremuloides). Cme-miR-10 is predicted to target a homolog of poplar genes POPTRDRAFT_755123 and POPTDRAFT_ 576281 (Table 3). The predicted target for Cme-miR-11 shows homology to protein with an F-box-containing domain and a putative ubiquitin protein ligase from castor bean (Ricinus communis). Qiao and associates (2009) reported that the stability of Arabidopsis ET-insensitive 2 (EIN2), a central regulator of ET response in plants, is modulated by F-box proteins ETP1 and ETP2. EIN2 in $\mathrm{Vat}^{+}$melon plants is upregulated after $6 \mathrm{~h}$ of A. gossypii feeding (Anstead et al. 2010). This indicates that F-box domain-containing proteins play a crucial role during aphid herbivory by fine tuning the ET pathway, and the miRNAs that target these proteins could be important in regulating ET responses in melon.

In summary, this study shows that miRNAs respond differentially to aphid infestation during a resistant and susceptible interaction. An overall increase in the smRNA population was detected in the resistant interaction. Also, the expression of conserved miRNAs was upregulated during different stages of the resistant interaction. Eighteen putative cucurbit-specific miRNAs and five melon-specific miRNA were identified in the libraries. Some of the conserved miRNAs presented in the library were found to target genes involved in biotic and abiotic stress responses.

\section{MATERIALS AND METHODS}

\section{Plant and insect growth conditions.}

The nearly isogenic AR 5 ( Vat $^{+}$, resistant) and PMR 5 (Vat ${ }^{-}$, susceptible) melon lines were grown in controlled conditions of $23^{\circ} \mathrm{C}, 60 \%$ relative humidity, and a photoperiod of $16 \mathrm{~h}$ of light and $8 \mathrm{~h}$ of darkness for 4 weeks until they reached the four-leaf stage. A. gossypii were reared and maintained on susceptible honeydew melon plants in a controlled growth chamber at $21^{\circ} \mathrm{C}$, with a photoperiod of $16 \mathrm{~h}$ of light and $8 \mathrm{~h}$ of darkness.

\section{Plant treatments.}

The third and fourth leaves from 4-week-old melon plants were used for aphid infestations. Each leaf was exposed to 50 apterous aphids confined in a $2.5-\mathrm{cm}$-diameter clip cage. A mix of aphids from different growth stages was used to model a natural crop infestation. Infestations were terminated after specific time periods: $2,4,6,8,10$, and $12 \mathrm{~h}$. After these periods, all aphids were gently brushed off the leaf tissue and a 2.5-cm leaf disk was excised. Leaf tissues collected from 2, 4, and $6 \mathrm{~h}$ of aphid infestation were pooled together (early-stage infestations), snap frozen in liquid nitrogen, and stored at $-80^{\circ} \mathrm{C}$. Similarly, leaf tissues collected from 8,10 , and $12 \mathrm{~h}$ of aphid infestation were combined (late-stage infestations) and stored at $-80^{\circ} \mathrm{C}$. Noninfested melon leaf tissues (clip cages without aphids) from identical time points were collected as controls and handled the same as the treatment tissues. All experiments were biologically independent and three independent replicates were used for each time point.

\section{Cloning of smRNA and Illumina sequencing.}

Total RNA was isolated by TRIzol reagent (Invitrogen) following the manufacturer's instructions for plants with high polysaccharide content. The RNA was enriched for LMW RNA by precipitation with $25 \%$ polyethylene glycol and $5 \mathrm{M}$ $\mathrm{NaCl}$ (Lu et al. 2007a). The LMW RNA quality was evaluated using an RNA600 Nano LabChip kit with the Agilent Bioanalyzer system (Agilent Biotechnologies, Santa Clara, CA, U.S.A.) and the concentration was measured with a Nanodrop spectrophotometer (Thermo Fisher Scientific, Wilmington, DE, U.S.A.).

LMW RNA $(50 \mu \mathrm{g})$ from control, early, and late infestations was separated in a $15 \%$ denaturing polyacrylamide gel. Size fractions of 20 to $24 \mathrm{nt}$ were excised and the RNA was extracted from the gel slices with $0.3 \mathrm{M} \mathrm{NaCl}$, followed by ethanol precipitation. The RNA was ligated to a 26-bp $5^{\prime}$ adapter, size selected on a polyacrylamide gel, and ligated to a $3^{\prime}$ adapter. The adapter sequences and PCR primers used in smRNA library preparation are provided in Supplementary Table S2.

First-strand cDNA synthesis was performed with the adapterligated smRNA template using the Superscript II reverse-transcriptase (Invitrogen) and a primer complementary to the $3^{\prime}$ adapter. The cDNA was used as a template for a low cycle PCR amplification (initial denaturation of $30 \mathrm{~s}$ at $98^{\circ} \mathrm{C} ; 15 \mathrm{cy}-$ cles of $98^{\circ} \mathrm{C}$ for $10 \mathrm{~s}, 60^{\circ} \mathrm{C}$ for $30 \mathrm{~s}$, and $72^{\circ} \mathrm{C}$ for $15 \mathrm{~s}$; followed by final extension at $72^{\circ} \mathrm{C}$ for $10 \mathrm{~min}$ ) to generate sufficient template for deep sequencing. The size-fractionated cDNA of approximately $100 \mathrm{bp}$ was gel eluted and subjected to Illumina GAII analyzer for deep sequencing.

\section{Bioinformatic analysis of the smRNA transcriptome.}

All Illumina sequencing data were initially converted to FASTA format from FASTQ. smRNA sequences were extracted from raw reads by matching them with the first $8 \mathrm{nt}$ of the $3^{\prime}$ adapter sequences. Based on the length of the mature miRNA and adapter length, sequences shorter than $18 \mathrm{nt}$ and 
greater than $30 \mathrm{nt}$ in length were removed. At this stage, the data were screened for redundant sequences. The remaining sequences were queried against ribosomal and transfer RNAs from Rfam and the Arabidopsis tRNA database. The matches to the rRNA and tRNA were discarded. Sequences having 10 or more counts were assigned to the unique sequence library. These sequences were then queried against melon coding RNA regions for perfect matches. Those sequences that matched to the sense strand were discarded as degraded mRNA; however, those matching to the anti-sense strand were retained and aligned against the miRBASE to identify the conserved miRNA (two mismatches allowed). All bioinformatic analyses were performed using custom-written PERL script. Bowtie, which is an ultrafast memory efficient short-read aligner (Langmead et al. 2009), was used to match the sequences to the pumpkin smRNA database, as well as the melon EST and cucumber 454 genome sequence databases. Unaligned sequences were potential candidates for novel melon miRNAs. Novel melon miRNAs were identified using the algorithm miRDeep (Friedlander et al. 2008). Because the complete genome sequence of melon was not available during the processing of the dataset, melon EST version 3.0 was used to find novel miRNAs.

\section{Expression profiling of conserved miRNAs in melon by qPCR.}

LMW-enriched RNA was used to study the differential expression pattern of conserved miRNA in the early and late aphid infestations. The miRNA expression was measured using a two-step process. In the first step, a stem-loop (RT) primer designed according to Chen and associates (2005) was hybridized to the miRNA and reverse transcribed in a pulsed RT reaction (Varkonyi-Gasic et al. 2007). In the second step, the RT reaction product was PCR amplified using a miRNAspecific forward primer and a universal primer (Supplementary Table S3) in real time with SYBR green chemistry using ABI 7500. The different members of the miRNA families expressed in the three libraries were aligned and an identical region was chosen for the design of the stem-loop (RT) primer and the miRNA-specific forward primer, allowing representative members of the miRNA to be amplified with near equal efficiency. The melon profilin gene (GenBank accession number AY879597) was used as housekeeping control and showed consistent expression during different stages of aphid herbivory (Anstead et al. 2010). The cycle threshold (Ct) values for profilin amplified from LMW RNA were comparable with the $\mathrm{Ct}$ values for profilin amplified from total RNA. Quantification of the relative changes in miRNA expression was performed using the method described by Pfaffl (2001). The data for relative quantities were converted to fold differences by logarithmic transformation to express the data as a normal distribution. The data were represented as averages of three measurements tstandard error. The comparisons of the miRNA expression levels between the three groups were performed using student's $t$ test $(P \leq 0.05)$. Bonferroni corrections were used to adjust for multiple comparisons.

\section{smRNA blots.}

Some of the miRNAs identified from the Illumina deep sequencing could not be verified by qPCR experiments. smRNA blots were performed to detect these miRNA and to confirm any discrepancy between the library data and the qPCR expression profiles. LMW RNA $(10 \mu \mathrm{g})$ was separated on a $15 \%$ denaturing polyacrylamide gel electrophoresis gel, electrophoretically transferred to $\mathrm{N}^{+}$hybond membrane (GE Healthcare, Piscataway, NJ, U.S.A.), and UV cross linked (Stratagene). Synthetic antisense DNA oligomers (IDT Inc.) were labeled at the $5^{\prime}$ end using $\left[\gamma^{32} \mathrm{P}\right]$-ATP (MP Biomedicals, Solon, $\mathrm{OH}$,
U.S.A.) and T4 Polynucleotide kinase (New England Biolabs, Ipswich, MA, U.S.A.) and used as probes. Probes were purified on a Sephadex G-25 column. Prehybridizations were carried out for $1 \mathrm{~h}$ using PerfectHyb Plus buffer (Sigma-Aldrich, St. Louis) at $37^{\circ} \mathrm{C}$ and hybridization was done for over $16 \mathrm{~h}$ using the same buffer. Hybridized membranes were washed twice at $52^{\circ} \mathrm{C}$ with $2 \times \mathrm{SSC}(1 \times \mathrm{SSC}$ is $0.15 \mathrm{M} \mathrm{NaCl}$ plus $0.015 \mathrm{M}$ sodium citrate) and $0.1 \%$ sodium dodecyl sulfate for $15 \mathrm{~min}$ each. The hybridized blots were exposed to phospoimager screens, viewed in TyphoonTrio (GE Healthcare), normalized with small nuclear RNA U6 oligonucleotide probe for smRNA gel blot analysis.

\section{Target prediction and validation.}

Conserved plant miRNA targets were previously validated in Arabidopsis thaliana and available on miRBASE. To verify whether the targets of the conserved miRNAs were also conserved in Cucumis melo, an in silico prediction analysis was performed using the sRNA target prediction tool at the $\mathrm{Cu}$ curbit Genomics Database. Some of the conserved miRNA had a related or completely new target gene. To further validate the results of the in silico analysis, an RNA ligase-mediated amplification of cDNA ends experiment was conducted for each putative cDNA target gene with the GeneRacer kit (Invitrogen, Carlsbad, CA, U.S.A.) according to the manufacturer's instructions.

\section{ACKNOWLEDGMENTS}

This research was supported by National Research Initiative of the United States Department of Agriculture National Institute of Food and Agriculture, grant number 2009-35302-30592. We thank the Recombinant DNA/Protein Resource facility at Oklahoma State University for providing access to their equipment and R. Al-ogaili for assistance with aphid maintenance.

\section{LITERATURE CITED}

Ahmadovichbozorov, T., Pandey, S. P., Dinh, S. T., Kim, S. G., Henrich, M., Gase, K., and Baldwin, I. T. 2012. Dicer-like proteins and plant herbivore interactions in Nicotiana attenuate. J. Integr. Plant Biol. 54:189-206.

Anstead, J., Samuel, P., Song, N., Wu, C., Thompson, G. A., and Goggin, F. L. 2010. Activation of ethylene-related genes in response to aphid feeding on resistant and susceptible melon and tomato plants. Entomol. Exp. Appl. 134:170-181.

Bohn, G. W., Kishaba, A. N., and Toba, H. H. 1972. Mechanisms of resistance to melon aphid in a muskmelon line. Hortic. Sci. 7:281-284.

Chen, C., Ridzon, D. A., Broomer, A. J., Zhou, Z., Lee, D. H., Nguyen, J. T., and Barbisin, M. 2005. Real-time quantification of microRNAs by stem-loop RT-PCR. Nucleic Acids Res. 33:179-188.

Chen, J. Q., Delobel, B., Rahbé, Y., and Sauvion, N. 1996. Biological and chemical characteristics of a genetic resistance of melon to the melon aphid. Entomol. Exp. Appl. 80:250-253.

Dogimont, C., Chovelon, V., Tual, S., Boissot, N., Rittener, V., Giovinazzo, N., and Bendahmane, A. 2008. Molecular diversity at the Vat/Pm-W resistance locus in melon. Pages 219-227 in: Cucurbitaceae 2008. Proc. IXth EUCARPIA Meet. Genet. Breeding of Cucurbitaceae. M. Pitrat, ed. INRA, Avignon, France.

Fahlgren, N., Howell, M. D., Kasschau, K. D., Chapman, E. J., Sullivan, C. M., Cumbie, J. S., Givan, S. A., Law, T. F., Grant, S. R., Dangla, J. L., and Carrington, J. C. 2007. High-throughput sequencing of Arabidopsis microRNAs: Evidence for frequent birth and death of MIRNA genes. PLoS ONE 2:e219. Published online.

Friedlander, M. R., Chen, W., Adamidi, C., Maaskola, J., Einspanier, R., Knespel, S., and Rajewsky, N. 2008. Discovering microRNAs from deep sequencing data using miRDeep. Nat. Biotechnol. 26:407-415.

Garzo, E., Soria, C., Gómez-Guillamón, M. L., and Fereres, A. 2002. Feeding behavior of Aphis gossypii on resistant accessions of different melon genotypes (Cucumis melo). Phytoparasitica 30:129-140.

Girousse, C., Moulia, B., Silk, W., and Bonnemain, J. L. 2005. Aphid infestation causes different changes in carbon and nitrogen allocation in alfalfa stems as well as different inhibitions of longitudinal and radial expansion. Plant Physiol. 137:1474-1484. 
Gonzalez-Ibeas, D., Blanca, J., Donaire, L., Saladie, M., Mascarell-Creus, A., Cano-Delgado, A., Garcia-Mas, J., Llave, C., and Aranda, M. 2011. Analysis of the melon (Cucumis melo) small RNAome by highthroughput pyrosequencing. BMC Genomics 12:e393.

He, X. F., Fang, Y., Feng, L., and Guo, H. 2008. Characterization of conserved and novel microRNAs and their targets, including a TuMVinduced TIR-NBS-LRR class R gene derived novel miRNA in Brassica. FEBS (Fed. Eur. Biochem. Soc.) Lett. 582:2445-2452.

Hewezi, T., Howe, P., Maier, T. R., and Baum, T. J. 2008. Arabidopsis small RNA and their targets during cyst nematode parasitism. Mol. Plant-Microbe Interact. 21:1622-1634.

Jones-Rhoades, M. W., and Bartel, D. P. 2004. Computational identification of plant microRNAs and their targets, including a stress-induced miRNA. Mol. Cell. 14:787-799.

Jones-Rhoades, M. W., Bartel, D., and Bartel, B. 2006. MicroRNAs and their regulatory roles in plants. Annu. Rev. Plant Biol. 57:19-53.

Kaloshian, I. 2004. Gene-for-gene disease resistance: Bridging insect pest and pathogen defense. J. Chem. Ecol. 30:2419-2438.

Katiyar-Agarwal, S., and Jin, H. 2010. Role of small RNAs in hostmicrobe interactions. Annu. Rev. Phytopathol. 48:225-246.

Kempema, L. A., Cui, X., Holzer, F. M., and Walling, L. L. 2007. Arabidopsis transcriptome changes in response to phloem-feeding silverleaf whitefly nymphs. Similarities and distinctions in responses to aphids. Plant Physiol. 143:849-865.

Kennedy, G. G., and Kishaba, A. N. 1977. Response of alate melon aphids to resistant and susceptible muskmelon lines. J. Econ. Entomol. 70:407410

Khan, J. A., Wang, Q., Sjölund, R. D., Schulz, A., and Thompson, G. A. 2007. An early nodulin like protein accumulates in the sieve element plasma membrane if Arabidopsis. Plant Physiol. 143:1576-1589.

Klinger, J., Powell, G., Thompson, G. A., and Isaacs, R. 1998. Phloem specific aphid resistance in Cucumis melo line AR 5: Effects on feeding behavior and performance of Aphis gossypii. Entomol. Exp. Appl. 86:79-88.

Kusnierczyk, A., Winge, P., Midelfart, H., Armbruster, W. S., Rossiter, J. T., and Bones, A. M. 2007. Transcriptional responses of Arabidopsis thaliana ecotypes with different glucosinolate profiles after attack by polyphagous Myzus persicae and oligophagous Brevicoryne brassicae. J. Exp. Bot. 58:2537-2552.

Langmead, B., Trapnell, C., Pop, M., and Salzberg, S. L. 2009. Ultrafast and memory-efficient alignment of short DNA sequences to the human genome. Genome Biol. 10:R25.

Li, Y., Zheng, Y., Addo-Quaye, C., Zhang, L., Saini, A., Jagadeeswaran, G., Axtell, M. J., Zhang, W., and Sunkar, R. 2010. Transcriptome-wide identification of microRNA targets in rice. Plant J. 62:742-759.

Lindow, M., and Krogh, A. 2005. Computational evidence for hundreds of non-conserved plant microRNAs. BMC Genomics 6:e119.

Lu, C., Meyers, B. C., and Green, P. 2007a. Construction of small RNA cDNA libraries for deep sequencing. Methods 43:110-117.

Lu, S. F., Sun, Y. H., Amerson, H., and Chiang, V. L., 2007b. MicroRNAs in loblolly pine (Pinus taeda L.) and their association with fusiform rust gall development. Plant J. 51:1077-1098.

Mallory, A., and Vaucheret, H. 2010. Form, function and regulation of Agronaute proteins. Plant Cell 22:3879-3889.

Martínez, G., Forment, J., Llave, C., Pallás, V., and Goméz, G. 2011. High-throughput sequencing, characterization and detection of new and conserved cucumber miRNAs. PLoS One 6:e19523. Published online.

McCreight, J. D., Kishaba, A. N., and Bohn, G. W. 1984. AR Hale's Best Jumbo, AR 5, and AR Topmark: Melon aphid resistant muskmelon breeding lines. Hortic. Sci. 19:309-310.

Micali, C., Göllner, K., Humphry, M., Consonni, C., and Panstruga, R. 2008. The Powdery Mildew Disease of Arabidopsis: A Paradigm for the Interaction Between Plants and Biotrophic Fungi. The Arabidopsis Book 6:e0115. The American Society of Plant Biologists, Rockville, MD, U.S.A. Published online.

Moran, P. J., Cheng, Y., Cassell, J. L., and Thompson, G.,A. 2002. Gene expression profiling of Arabidopsis thaliana in compatible plant-aphid interactions. Arch. Insect Biochem. 51:182-203.

Navarro, L., Dunoyer, P., Jay, F., Arnold, B., Dharmasiri, N., Estelle, M. Vionnet, O., and Jones, J. D. G. 2006. A plant miRNA contributing to antibacterial resistance by repression auxin signaling. Science 321:436439.

Noordermeer, M. A., Veldink, G. A., and Vliegenthart, J. F. G. 2001. Fatty acid hydroperoxide lyase: A plant cytochrome $\mathrm{P} 450$ enzyme involved in wound healing and pest resistance. Chembiochem 2:494-504.

Pandey, S. P., Shahi, P., Gase, K., and Baldwin, I. T. 2008. Herbivory induced small-RNA induced transcriptome and phytohormone signaling in Nicotiana attenuata. Proc. Natl. Acad. Sci. U.S.A. 105:4559-4564.

Pegadaraju, V., Knepper, C., Reese, J and Shah, J. 2005. Premature leaf senescence modulated by the Arabidopsis PHYTOALEXIN DEFICIENT4 gene is associated with defense against the phloem-feeding green peach aphid. Plant Physiol. 139:1927-1934.

Pfaffl, M. 2001. A new mathematical model for relative quantification in real-time RT-PCR. Nucleic Acids Res. 29:2002-2007.

Qiao, H., Chang, K. N., Yazaki, J., and Ecker, J. R. 2009. Interplay between ethylene, ETP1/ETP2 F-box proteins, and degradation of EIN2 triggers ethylene responses in Arabidopsis. Genes Dev. 23:512.

Rhoades, M. W., Reinhart, B. J., Lim, L. P., Burge, C. B., Bartel, B., and Bartel. D. P. 2002. Prediction of plant microRNA targets. Cell 110:513520.

Smith, C. M., and Boyko, E. V. 2007. The molecular basis of plant resistance and defense responses to aphid feeding: Current status. Entomol. Exp. Appl. 221:1-6.

Sunkar, R. 2010. MicroRNAs with macro-effects on plant stress responses. Semin. Cell Dev. Biol. 21:805-811.

Sunkar, R., Kapoor, A., and Zhu, J. 2006. Posttranscriptional induction of two $\mathrm{Cu} / \mathrm{Zn}$ superoxide dismutase genes in Arabidopsis is mediated by downregulation of miR398 and important for oxidative stress tolerance. Plant Cell 18:2051-2065.

Varkonyi-Gasic, E., Wu, R., Wood, M., Walton, E. F., and Hellens, R. P. 2007. Protocol: A highly sensitive RT-PCR method for detection and quantification of microRNAs. Plant Methods 3:12.

Vidal, E. A., Araus, V., Lu, C., Parry, G., Green, P. J., Coruzzi, G. M., and Gutiérrez, R. A. 2010. Nitrate-responsive miR393/AFB3 regulatory module controls root system architecture in Arabidopsis thaliana. Proc. Natl. Acad. Sci. U.S.A. 107:4477-4482.

Yang, X., Zhang, H., and Li, L. 2011. Global analysis of gene-level miRNA expression in Arabidopsis using deep sequencing data. Genomics 98:40-46

Yi, H., Galant, A., Ravilious, G. E., Preuss, M. L., and Jez, J. M. 2010. Sensing sulfur conditions: Simple to complex protein regulatory mechanisms in plant thiol metabolism. Mol. Plant 3:269-279.

Zechmann, B., Zelený, F., Wonisch, A., and Zelená, E. 2009. Aphid infestation affects subcellular glutathione and cysteine contents in Beta vulgaris. Belg. J. Bot. 142:50-59.

Zhu, J. 2008. Reconstituting plant miRNA biogenesis. Proc. Natl. Acad. Sci. U.S.A. 105:9851-9852.

\section{AUTHOR-RECOMMENDED INTERNET RESOURCES}

Cucurbit genomics database:

www.icugi.org/cgi-bin/ICuGI/genome/cuke.cgi

The Genomic tRNA database: lowelab.ucsc.edu/GtRNAdb/Athal

miRBase: The microRNA database: www.mirbase.org

Sanger Institute Rfam database: www.sanger.ac.uk/resources/databases 